Objectives: The goal of the study was to assess the relationship of NLR, PLR, MLR with the conventional disease activity markers (ESR, CRP, DAS28, TJC, SJC) and results of joints US, in patients with RA. Methods: The study was conducted in the consecutive 72 patients with RA (60 women, 12 men), with the mean (SD) age 53.4 (8.9) and disease duration 16.8 (10.3) years. The following procedures were assessed for all patients: joint counts,DAS28, complete blood cell counts, ESR, CRP, US of 24 small joints.

Results: The mean (SD) values of hematological markers were as follows: NLR 3.24 (2.7) (range 0.2-13.96); PLR 193.26 (88.3) (range 71.2560.8); MLR 0.3 (0.16) (range 0.06-0.87). The mean values of NLR and PLR were significantly lower in patients with low (DAS28 $\leq 3.2$ ) vs moderate/high (DAS28 >3.2) disease activity; respectively NLR 2.4 (1.7) vs 4.0 (3.2), $\mathrm{p}=0.004$; PLR 168.7 (58.0) vs 215.8 (104.9), $\mathrm{p}=0.4$. Hematological markers of systemic inflammation were significantly, positively correlated with clinical, laboratory and US markers of the disease activity. Significant correlations were found between: NLR and DAS28, TJC, SJC, ESR, CRP, vascularity of synovium in US; PLR and DAS28, TJC, SJC, ESR, CRP, vascularity and hypertrophy of synovium in US; MLR and DAS28, SJC, ESR, CRP, vascularity and hypertrophy of synovium in US. In multiple regression test significant correlations were confirmed for: NLR and SJC $(p=0.008)$, ESR $(p<0.001), \operatorname{CRP}(p<00.1)$, vascularity of synovium in US $(p=0.02)$; PLR and ESR $(p=0.03)$, CRP $(p=0.03)$; MLR and DAS28 $(p=0.04)$, ESR $(p<0.001)$, hypertrophy of synovium in US $(p=0.02)$.

Conclusion: The results of the study suggest that hematological markers of systemic inflammation (NLR, PLR, MLR) may serve as reliable, inexpensive and effective measure of inflammation in RA. The strong association with US imaging is an attractive result because of its predictive value and usefulness in clinical practice

Disclosure of Interests: Bozena Targonska-Stepniak Speakers bureau: Sandoz, Berlin-Chemie, Robert Zwolak: None declared, Mariusz Piotrowski: None declared, Maria Majdan Speakers bureau: MSD, UCB, Abbvie, Roche

DOI:10.1136/annrheumdis-2019-eular.2307

\section{AB0285 PREDICTORS OF UNACCEPTABLE PAIN, AND UNACCEPTABLE PAIN WITH LOW INFLAMMATION, IN EARLY RHEUMATOID ARTHRITIS}

Anna Eberhard ${ }^{1}$, Tor Olofsson ${ }^{2,3}$, Lennart T.H. Jacobsson ${ }^{4}$, Carl Turesson $^{1,3}$ ${ }^{1}$ Lund University, Rheumatology, Department of Clinical Sciences, Malmö, Malmö, Sweden; ${ }^{2}$ Lund University, Rheumatology, Department of Clinical Sciences, Lund, Lund, Sweden; ${ }^{3}$ Skåne University Hospital, Department of Rheumatology, Malmö, Sweden; ${ }^{4}$ Sahlgrenska Academy at Gothenburg University, Department of Rheumatology and Inflammation Research, Gothenburg, Sweden

Background: Pain is a major symptom in many patients with rheumatoid arthritis (RA). In early RA, pain is usually due to active synovitis, but over the disease course some patients experience pain without elevated laboratory markers of inflammation.

Objectives: To investigate predictors of unacceptable pain, and unacceptable pain with low inflammation, in patients with early RA.

Methods: Consecutive patients with early RA (symptom duration $<12$ months), recruited in 1995-2005 from a defined area, were followed through 5 years. Patients were managed according to usual care, with no pre-specified treatment protocol. Pain was assessed using a visual analogue scale (VAS; 0-100 mm). Unacceptable pain was defined as VAS pain $>40$ based on the patient acceptable symptom state (PASS) (1), and low inflammation as CRP $<10 \mathrm{mg} / \mathrm{l}$ (2). Baseline predictors of unacceptable pain, and of unacceptable pain with low inflammation, were evaluated using logistic regression analysis.

Results: A total of 233 patients with early RA (73\% female, $57 \%$ antiCCP positive, mean age 60 years, median symptom duration 7 months) were included. Of these, 179 attended the 5-year follow-up. At 5 years, $34 \%$ had unacceptable pain, and $23 \%$ had unacceptable pain with low inflammation. High VAS scores for pain and patient's global assessment (PGA) at baseline were associated with unacceptable pain at 5 years (Table). There was a negative association between baseline swollen joint count (SJC28) and unacceptable pain at the 5 year follow-up. In multivariate logistic regression analysis including VAS PGA and SJC28, both had an impact on unacceptable pain after 5 years (adjusted odds ratios per standard deviation (SD), with $95 \%$ Cl 1.78 (1.26-2.52) and 0.61 (0.42-0.89), respectively). Anti-CCP positive patients were significantly less likely to experience unacceptable pain with low inflammation at 5 years (Table).
Table 1.

\begin{tabular}{|c|c|c|c|c|}
\hline \multirow[b]{2}{*}{ Variable } & \multicolumn{2}{|c|}{ Unacceptable pain } & \multicolumn{2}{|c|}{ Unacceptable pain with low inflammation } \\
\hline & Odds ratio & $95 \% \mathrm{CI}$ & Odds ratio & $95 \%$ CI \\
\hline Female & 1.24 & $0.62-2.47$ & 1.87 & $0.80-4.40$ \\
\hline RF positive & 0.88 & $0.46-1.67$ & 0.54 & $0.26-1.09$ \\
\hline Anti-CCP positive & 0.77 & $0.39-1.49$ & 0.50 & $0.22-0.98$ \\
\hline Erosion & 0.62 & $0.26-1.49$ & 0.62 & $0.22-1.72$ \\
\hline Age & 1.09 & $0.80-1.49$ & 0.97 & $0.68-1.38$ \\
\hline Symptom duration & 1.28 & $0.93-1.76$ & 1.19 & $0.83-1.71$ \\
\hline VAS pain & 1.40 & $1.02-1.91$ & 1.34 & $0.94-1.92$ \\
\hline DAS28 & 1.03 & $0.76-1.41$ & 1.00 & $0.70-1.42$ \\
\hline SJC28 & 0.71 & $0.51-0.99$ & 0.70 & $0.47-1.03$ \\
\hline TJC28 & 0.98 & $0.71-1.35$ & 1.04 & $0.72-1.50$ \\
\hline $\mathrm{HAQ}$ & 1.01 & $0.74-1.37$ & 1.01 & $0.72-1.43$ \\
\hline CRP $>9 \mathrm{mg} / 1$ & 0.64 & $0.29-1.41$ & 0.62 & $0.23-1.72$ \\
\hline ESR & 0.79 & $0.57-1.09$ & 0.74 & $0.50-1.11$ \\
\hline VAS PGA & 1.60 & $1.16-2.21$ & 1.39 & $0.98-2.00$ \\
\hline
\end{tabular}

Odds ratios per SD for continuous variables

Conclusion: More than $1 / 3$ of early RA patients experienced unacceptable pain after 5 years. Extensive synovitis in early RA was associated with a reduced risk of unacceptable pain at 5 years, likely due to positive effects of treatment on inflammation related pain. Non-inflammatory pain may be a greater long term problem in anti-CCP negative patients.

\section{REFERENCES}

[1] Tubach, et al. Arthritis Care Res 2012; 64: 1699-707

[2] Lourdudoss, et al. Arthritis Care Res 2018; 70: 205-12.

Disclosure of Interests: Anna Eberhard: None declared, Tor Olofsson: None declared, Lennart T.H. Jacobsson Consultant for: LJ has received lecture and consulting fees from Pfizer, Abbvie, Novartis, Eli-Lily and Janssen, Carl Turesson: None declared

DOI: 10.1136/annrheumdis-2019-eular.3627

\section{$\mathrm{AB} 0286$ \\ STATINS TO PREVENT RHEUMATOID ARTHRITIS (STAPRA TRIAL): CHALLENGES IN RECRUITMENT AND RETENTION}

Laurette van Boheemen ${ }^{1}$, S.A. Turk ${ }^{1}$, van Beers - Tas ${ }^{1}$, W.H. Bos ${ }^{2}$, E.N. Griep ${ }^{3}$, A. M. van Sij ${ }^{1}$, Maarten Boers ${ }^{4}$, Michael Nurmohamed ${ }^{4}$, Dirkjan van Schaardenburg ${ }^{5}$. ${ }^{1}$ ARC Reade, Amsterdam, Netherlands; ${ }^{2}$ Sint Maartenskliniek, Nijmegen, Netherlands; ${ }^{3}$ Antonius Ziekenhuis, Sneek, Netherlands; ${ }^{4} A R C$ VUmc, Amsterdam, Netherlands; ${ }^{5}$ ARC AMC, Amsterdam, Netherlands

Background: Primary prevention may be possible in subjects at high risk to develop rheumatoid arthritis (RA). We designed a placebo controlled randomized trial to investigate if atorvastatin can halt RA development in persons at risk for this disease (STAPRA, Netherlands Trial Register NTR5265). A statin was chosen because these drugs reduce disease activity in RA (1), hyperlipidemia patients on statins have a lower risk for developing RA (2) and dyslipidemia increased the risk for RA in a seropositive arthralgia cohort (3). We assumed that high risk-subjects would be attracted to this trial. However, we experienced severe difficulties with patient inclusion and treatment adherence.

Objectives: To explore difficulties with patient recruitment and retention.

Methods: The STAPRA study is a multicenter, 3-year, randomized, placebo controlled, double-blind clinical trial to assess the efficacy of atorvastatin $40 \mathrm{mg}$ daily in delaying or preventing RA development in persons at high risk, defined by the presence of arthralgia and the presence of high titers of anti-citrullinated protein antibody (ACPA) or presence of both ACPA and rheumatoid factor (RF). Eligible participants were $\geq 18$ years, did not use lipid lowering agents and had no synovitis. Five centers participated. Our goal was to recruit 220 study subjects based on an expected risk reduction of $21 \%$. The unexpected low recruitment rate prompted us to evaluate the reasons to decline participation or to discontinue the study drug prematurely.

Results: Details were available from the initiating center (Reade) and 1 participating center (Sint Maartenskliniek (SMK)). During a period of 36 months, 164 eligible patients were asked to participate of whom 58 patients $(35 \%)$ consented. Most common reasons to decline were: unwillingness to use study medication (49\%) and the feeling that participation was too time-consuming (14\%). Fifty-four patients were randomized since 4 failed screening due to hyperlipidemia or seronegativity on repeated testing. Currently, 11 participants $(20 \%)$ have developed arthritis. Twelve 